\title{
The effects of strength training and raloxifene on bone health in aging ovariectomized rats
}

\author{
Camila Tami Stringhetta-Garcia ${ }^{\mathrm{a}, *}$, Monique Patrício Singulani ${ }^{\mathrm{a}}$, Leandro Figueiredo Santos ${ }^{\mathrm{a}}$, \\ Mário Jefferson Quirino Louzada ${ }^{\mathrm{a}, \mathrm{b}}$, Ana Cláudia Stevanato Nakamune ${ }^{\mathrm{a}, \mathrm{c}}$, Antonio Hernandes Chaves-Neto ${ }^{\mathrm{c}}$, \\ Ana Cláudia Rossi ${ }^{\mathrm{d}}$, Edilson Ervolino ${ }^{\mathrm{c}}$, Rita Cássia Menegati Dornelles ${ }^{\mathrm{a}, \mathrm{c}}$ \\ a Programa Multicêntrico de Pós-Graduação em Ciências Fisiológicas \\ ${ }^{\mathrm{b}}$ Faculdade de Medicina Veterinária de Araçatuba, UNESP - Univ Estadual Paulista, Campus de Araçatuba, Departamento de Apoio, Produção e Saúde Animal \\ c Faculdade de Odontologia de Araçatuba, UNESP - Univ Estadual Paulista, Campus de Araçatuba, Departamento de Ciências Básicas \\ ${ }^{\mathrm{d}}$ Faculdade de Odontologia de Piracicaba, UNICAMP - Univ de Campinas, Campus de Piracicaba, Departamento de Morfologia
}

\section{A R T I C L E I N F O}

\section{Article history:}

Received 14 April 2015

Revised 9 November 2015

Accepted 28 November 2015

Available online 23 January 2016

\section{Keywords:}

Aging

Osteoporosis

Strength training

Raloxifene

Bone microarchitecture

\begin{abstract}
A B S T R A C T
The aim of this study was to investigate the effects of strength training (ST) and raloxifene (Ral), alone or in combination, on the prevention of bone loss in an aging estrogen-deficient rat model. Aging Wistar female rats were ovariectomized at 14 months and allocated to four groups: (1) non-trained and treated with vehicle, NT-Veh; (2) strength training and treated with vehicle, ST-Veh; (3) non-trained and treated with raloxifene, NT-Ral; and (4) strength training and treated with raloxifene, ST-Ral. ST was performed on a ladder three times per week and Ral was administered daily by gavage $(1 \mathrm{mg} / \mathrm{kg} / \mathrm{day})$, both for 120 days. Areal bone mineral density (aBMD), strength, microarchitecture, and biomarkers (osteocalcin, OCN; osteoprotegerin, OPG; and tartrateresistant acid phosphatase, TRAP) were assessed. Immunohistochemistry was performed for runt-related transcription factor 2 (RUNX2), osterix (OSX), OCN, OPG, TRAP, and receptor activator of nuclear factor kappa-B ligand (RANKL). The rats that performed ST (ST-Veh) or were treated with Ral (NT-Ral) showed significant improvements in $\operatorname{aBMD}(p=0.001$ and 0.004$)$, bone strength $(p=0.001)$, and bone microarchitecture, such as BV/ TV (\%) $(p=0.001), \mathrm{BS} / \mathrm{TV}\left(\mathrm{mm}^{2} / \mathrm{mm}^{3}\right)(p=0.023$ and 0.002$)$, Conn.Dn $\left(1 / \mathrm{mm}^{3}\right)(p=0.001), \mathrm{Tb} . \mathrm{N}(1 / \mathrm{mm})$ $(p=0.012$ and 0.011$)$, Tb.Th $(1 / \mathrm{mm})(p=0.001)$, SMI $(\mathrm{p}=0.001$ and 0.002$)$, Tb.Sp $(p=0.001)$, and DA $(p=0.002$ and 0.007$)$; there was also a significant decrease in plasma levels of OCN $(p=0.001$ and 0.002$)$ and OPG ( $p=0.003$ and 0.014$)$, compared with animals in the NT-Veh group. Ral, with or without ST, promoted an increased immunolabeling pattern for RUNX2 $(p=0.0105$ and $p=0.0006)$ and OSX ( $p=0.0105)$, but a reduced immunolabeling pattern for TRAP $(p=0.0056)$ and RANKL $(p=0.033$ and 0.004$)$. ST increased the immunolabeling pattern for RUNX2 $(p=0.0105)$, and association with Ral resulted in an increased immunolabeling pattern for OPG $(p=0.0034)$ and OCN $(p=0.0024)$. In summary, ST and Ral administration in aged, estrogen-deficient Wistar female rats is associated with a decrease in bone turnover marker plasma levels, increased activity of cells that promote osteoblastogenesis, and decreased activity of cells that promote osteoclastogenesis; these are correlated with higher aBMD, bone strength, and bone microarchitecture at the femoral neck. The results indicate that strength training and Ral are potential tools to reduce the risk of fractures at clinically relevant sites.
\end{abstract}

(c) 2016 Elsevier Inc. All rights reserved.

\section{Introduction}

Osteoporosis is a bone disease characterized by increased risk of fracture. Its prevalence is increasing as a result of longer life expectancies and population aging; consequently, it is responsible for a rise in

\footnotetext{
* Corresponding author at: Department of Basic Sciences, Araçatuba Dental School, UNESP, Rodovia Marechal Rondon, km 527, CEP 16018-805, Araçatuba, SP, Brazil.

E-mail address: camilatami@foa.unesp.br (C.T. Stringhetta-Garcia).
}

social and public health problems [1,2]. Osteoporosis arises from changes in bone homeostasis, which result in impairments to the remodeling cycle [3]. Hip fractures are one of the most common types of fracture that affect people with osteoporosis, and are associated with a higher level of disability and health care costs than all other osteoporotic fractures $[4,5]$. Fourteen percent of the total number of fractures in the USA are femoral neck fractures, which account for $72 \%$ of the total cost of treating osteoporotic fractures [6].

Among local and systemic factors that contribute to an imbalance in the activity of bone cells, estrogen has an important role to play in bone health as a result of both direct and indirect activity [7]. Postmenopausal 
women have an increased risk of fragility fractures related to changes in hormonal levels [8]; these changes result in a decrease in bone mineral density (BMD), due to an increase in bone resorption relative to bone formation [9]. Preventive and therapeutic interventions for maintaining bone health are therefore extremely important given the impact on functional independence and quality of life in older people. In recent years, several studies have identified pathways involved in the maintenance and formation of bone that can be used for targeting drug therapy, such as the use of anabolic agents (parathyroid hormone) [10], and antiresorptive treatments, such as immunological (RANKL antibody) [11], hormone replacement [12], and bisphosphonate therapy [13].

Raloxifene (Ral), a selective estrogen receptor modulator (SERM), is an example of a class of pharmacological compounds with beneficial effects on bone tissue [14] and that lower the risk of breast cancer [15]. Clinical studies have demonstrated that Ral prevents bone loss and lowers the risk of fracture by modulating bone turnover [16]. Ral has been described as an antiresorptive drug, but the presence of estrogen receptors in osteoblasts and bone marrow stromal cells suggests that Ral can have a direct role in the regulation of osteoblast lineage cells as well as an osteoblast stimulatory role $[17,18]$. However, the mechanism by which this SERM exerts its effect on bone cells has yet to be fully elucidated [17], and no in vivo study has documented an effect of Ral on osteoblasts.

Strength training (ST) has been proposed as an alternative to drug therapy in the prevention and treatment of osteoporosis, as a means of preventing deterioration of bone mass. ST exercises are known to increase bone strength and bone mass [19], and improve bone microarchitecture [20], whereas a sedentary lifestyle has been associated with increased bone resorption [21]. Mechanical loading also influences a range of tissues, including muscle, tendons, and ligaments, and represents a means of protecting skeletal integrity in a nonpharmacological fashion [22]. In the postmenopausal period, there is decreased activity of estrogen receptor $\alpha$ in bone cells, with a concurrent decline in the responsiveness of bone to mechanical load, which further exacerbates deterioration of bone quality [23-25]. This is a possible explanation for bone loss in postmenopausal women in amounts comparable to that associated with disuse [26].

Considering bone fragility in old age, it is essential to propose interventions that can minimize bone loss and validate preventive measures for primary osteoporosis. Thus, the aim of this study was to investigate the effects of ST and Ral, alone or in combination, on the prevention of bone loss in an aged estrogen-deficient rat model.

\section{Material and methods}

\subsection{Animals}

All animal procedures were approved (Process number 001,3972010) by the Institutional Animal Care and Use Committee of the Faculty of Dentistry (Univ. Estadual Paulista - UNESP, Araçatuba, SP, Brazil) and complied with the Guide for Care and Use of Laboratory Animals.

Female Wistar rats aged 13-18 months were obtained from the central animal facility of the Faculty of Dentistry of Araçatuba, and were housed at $22{ }^{\circ} \mathrm{C}\left( \pm 2{ }^{\circ} \mathrm{C}\right)$ under a $12: 12 \mathrm{~h}$ light:dark cycle. The animals were allowed free access to water and a commercial pellet diet (Presence® Ratos e Camundongos, Paulínia, SP, Brazil). All female rats were multiparous as this was an inclusion criterion. The reproductive life of these animals began on average at 2 to 8 months of age, reaching 3 to 4 pregnancies. The general health of the rats was monitored on a daily basis and the estrus cycle was checked during the 13th month, to determine acyclicity. In female rats, the incidence of regular estrous cyclicity decreases progressively during aging and their estrous cycles tend to become irregular, usually with prolonged estrus and diestrus, characterizing peri-estropause. This period is similar to perimenopause in women [27-29]. Thus, rats were ovariectomized to decrease estradiol plasma concentrations even more, similar to what occurs at menopause, and to study changes at the beginning of this new phase in naturally aging female rats.

At 14 months of age, acyclic animals were submitted to a bilateral ovariectomy [30]. For the ovariectomy procedure, animals were anesthetized with ketamine (Vetaset ${ }^{\circledR}$, Fort Dodge Saude Animal Ltda, Brazil) at a dose of $50 \mathrm{mg} / \mathrm{kg}$ of body weight, in combination with xylazine (Coopazine, Coopers Brazil Ltda) at a dose of $25 \mathrm{mg} / \mathrm{kg}$ of body weight, by intraperitoneal injection. Incisions were made at the side of the abdomen to enable removal of the ovaries. Animals were sutured and received a prophylactic intramuscular dose of antibiotic ( $1 \mathrm{~mL} / \mathrm{kg}$; Veterinary Pentabiotic; Fort Dodge Animal Health Ltd.). Ten days after surgery, ovariectomized (OVX) rats were randomly assigned to one of four groups: (1) non-trained and treated with vehicle (NT-Veh, $\mathrm{n}=10$ ), (2) strength training and treated with vehicle (ST-Veh, $\mathrm{n}=10$ ), (3) non-trained and treated with raloxifene (NT-Ral, $\mathrm{n}=10$ ), and (4) strength training plus raloxifene (STRal, $\mathrm{n}=10$ ). After 120 days, the animals were sacrificed with anesthetic overdose; the uterus was collected and weighed to verify the success of ovariectomy.

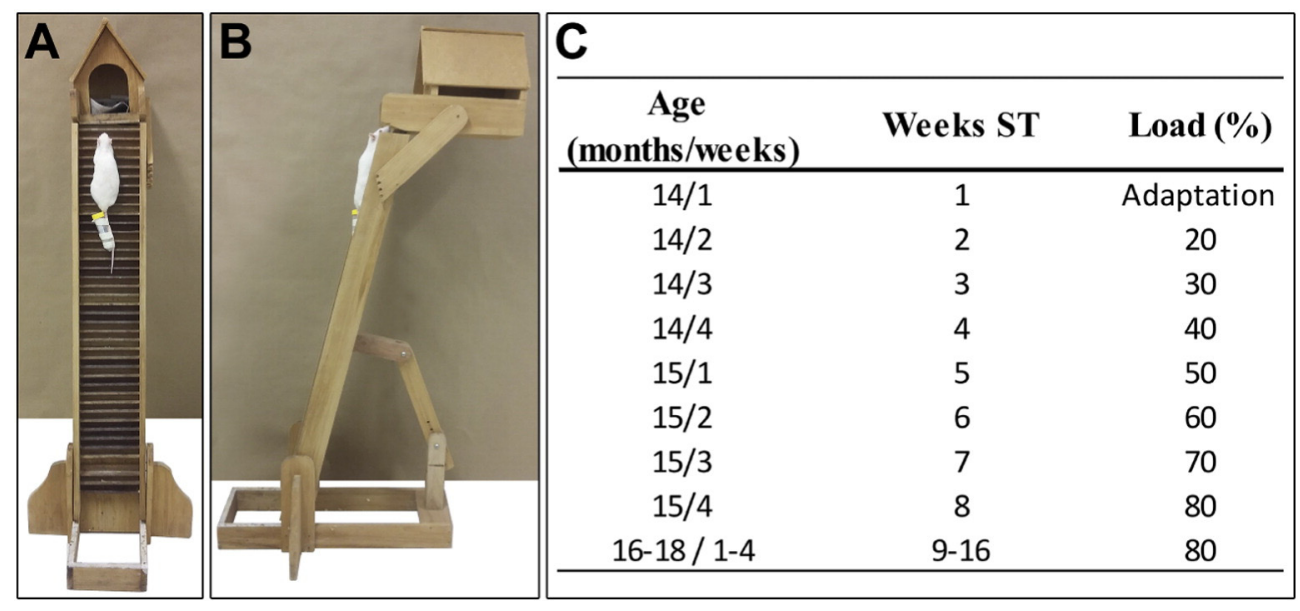

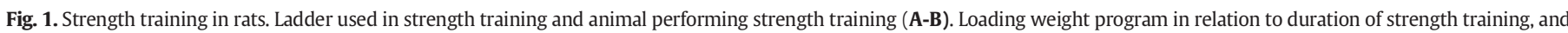
percentage of loading weight $(\mathbf{C})$. 


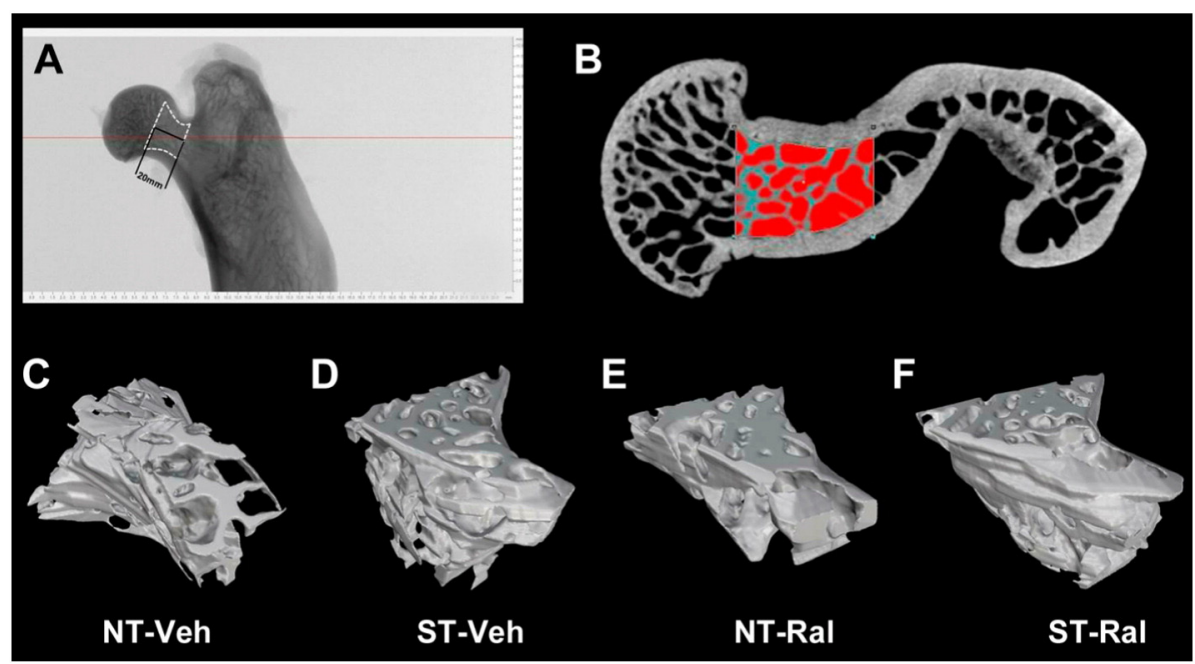

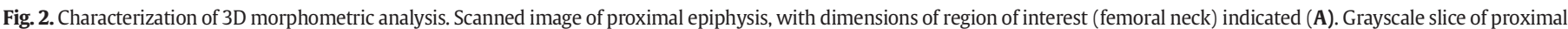

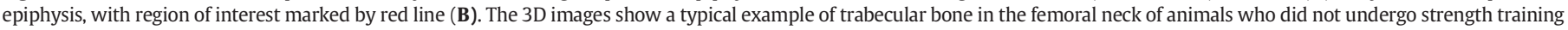

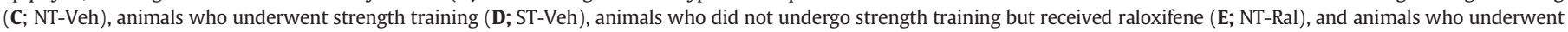
strength training and received raloxifene (F; ST-Ral).

\subsection{Raloxifene and vehicle administration}

Ten days after the ovariectomy, animals in the NT-Ral and ST-Ral groups received $1 \mathrm{mg} / \mathrm{kg}$ /day Ral (Sigma Aldrich, Munich, Germany) in $0.3 \mathrm{~mL}$ of physiological saline solution [31,32] administered daily by gavage for 120 days. Animals in the other treatment groups received physiological saline solution $(0.3 \mathrm{~mL})$ daily by gavage for the same period.

\subsection{Strength training}

ST in ST-Veh and ST-Ral groups was carried out by climbing sessions on a ladder $\left(1.13 \times 0.18 \mathrm{~m}, 2 \mathrm{~cm}\right.$ grid, $80^{\circ}$ angle, with a $20 \times 20 \times 20 \mathrm{~cm}$ diameter resting area at the top; Fig. $1 \mathrm{~A}$ and $\mathrm{B}$ ) three times per week [33]. Ten days after the ovariectomy, the animals underwent 1 week of acclimation. Subsequently, the maximum strength of each animal was evaluated with the use of two weighted tubes (steel spheres) attached to the tail. The maximum strength was measured by attaching a $50 \mathrm{~g}$ weight to the tail; this load was increased by $20 \mathrm{~g}$ for each climb until concentric failure (i.e., when the rat was no longer able to climb the ladder); the previous load up to this point was considered the maximum strength. ST began with $20 \%$ maximum strength, and the load was increased by $10 \%$ each week to reach $80 \%$ maximum strength, which was maintained until the end of the experiment (Fig. 1C). Monthly maximum force tests were performed to readjust

Table 1

Final (18 months) body weight, uterus weight of animals at time of necropsy, and estradiol plasma concentration.

\begin{tabular}{llll}
\hline Group & Final Weight $(\mathrm{g})$ & Uterus Weight $(\mathrm{g})$ & Estradiol $(\mathrm{pg} / \mathrm{mL})$ \\
\hline NT-Veh & $368.3 \pm 22.41$ & $0.084 \pm 0.012$ & $69.21 \pm 3.66$ \\
ST-Veh & $392.0 \pm 18.74$ & $0.079 \pm 0.006$ & $66.81 \pm 3.81$ \\
NT-Ral & $351.5 \pm 8.98$ & $0.091 \pm 0.006$ & $77.33 \pm 5.18$ \\
ST-Ral & $356.9 \pm 8.80$ & $0.094 \pm 0.010$ & $70.08 \pm 4.26$ \\
\hline
\end{tabular}

Final body weight, uterine weight/100 g of body weight, and estradiol plasma concentration of aged OVX female Wistar rats with or without strength training, treated for 16 weeks with vehicle or raloxifene. Values represent the mean \pm SEM.

NT-Veh $=$ non-trained and treated with vehicle; ST-Veh = strength training and treated with vehicle; NT-Ral = non-trained and treated with raloxifene; ST-Ral = strength training and treated with raloxifene. the load. Increases in load were made gradually, and tailored according to the characteristics of individual animals. Increments in load took into account the age of animals and the maximum force test but not their body weight. The ST protocol consisted of 3 sessions per week for 120 days. Each session consisted of 4 sets of 5 climbs and a rest period of 2 min between each set. No animals were excluded due to physical injuries or disorders in the estrous cycle.

2.4. Measurement of plasma levels of osteocalcin, osteoprotegerin, and tartrate-resistant acid phosphatase

After 120 days of treatment, all animals were anesthetized with ketamine at a dose of $80 \mathrm{mg} / \mathrm{kg}$ of body weight combined with xylazine at dose of $10 \mathrm{mg} / \mathrm{kg}$ of body weight intraperitoneally. Blood $(3 \mathrm{~mL})$ was collected from the external jugular vein and placed into a centrifuge tube. The plasma was separated by centrifugation at $2256 \mathrm{~g}$ for $15 \mathrm{~min}$ at $4{ }^{\circ} \mathrm{C}$, and stored at $-20{ }^{\circ} \mathrm{C}$. Levels of plasma osteocalcin (OCNE90471Ra), osteoprotegerin (OPG-E90108Ra), and tartrate-resistant acid phosphatase (TRAP-E90902Ra) were determined by employing a rat-specific quantitative sandwich enzyme-linked immunosorbent assay (ELISA, Uscn Life Science Inc., Wuhan, China).

\subsection{Measurement of plasma levels of estradiol}

The estradiol levels were measured using a commercial radioimmunoassay kit (Estradiol Maia, Adaltis Italia S.p.A., Casalecchio di Reno, BO, Italy). All samples were assayed in duplicate and in the same assay to avoid inter-assay error. The minimum detectable dose of estradiol was $5.0 \mathrm{pg} / \mathrm{mL}$, and the intra-assay value was $3.9 \%$.

\subsection{Bone mineral density measurements}

Animals were euthanized with an overdose of anesthetic. Femurs were extracted, cleaned with soft tissue, kept frozen at $-20{ }^{\circ} \mathrm{C}$, and slowly thawed and maintained immersed in saline solution until testing. For the areal bone mineral density (aBMD), femurs were thawed and positioned in the frontal plane and anterior posterior view on the scanner table, all oriented the same way, and were fully scanned in a bowl with $2 \mathrm{~cm}$ of water, according to manufacturer instructions. The aBMD of femurs was assessed using dual energy X-ray absorptiometry 

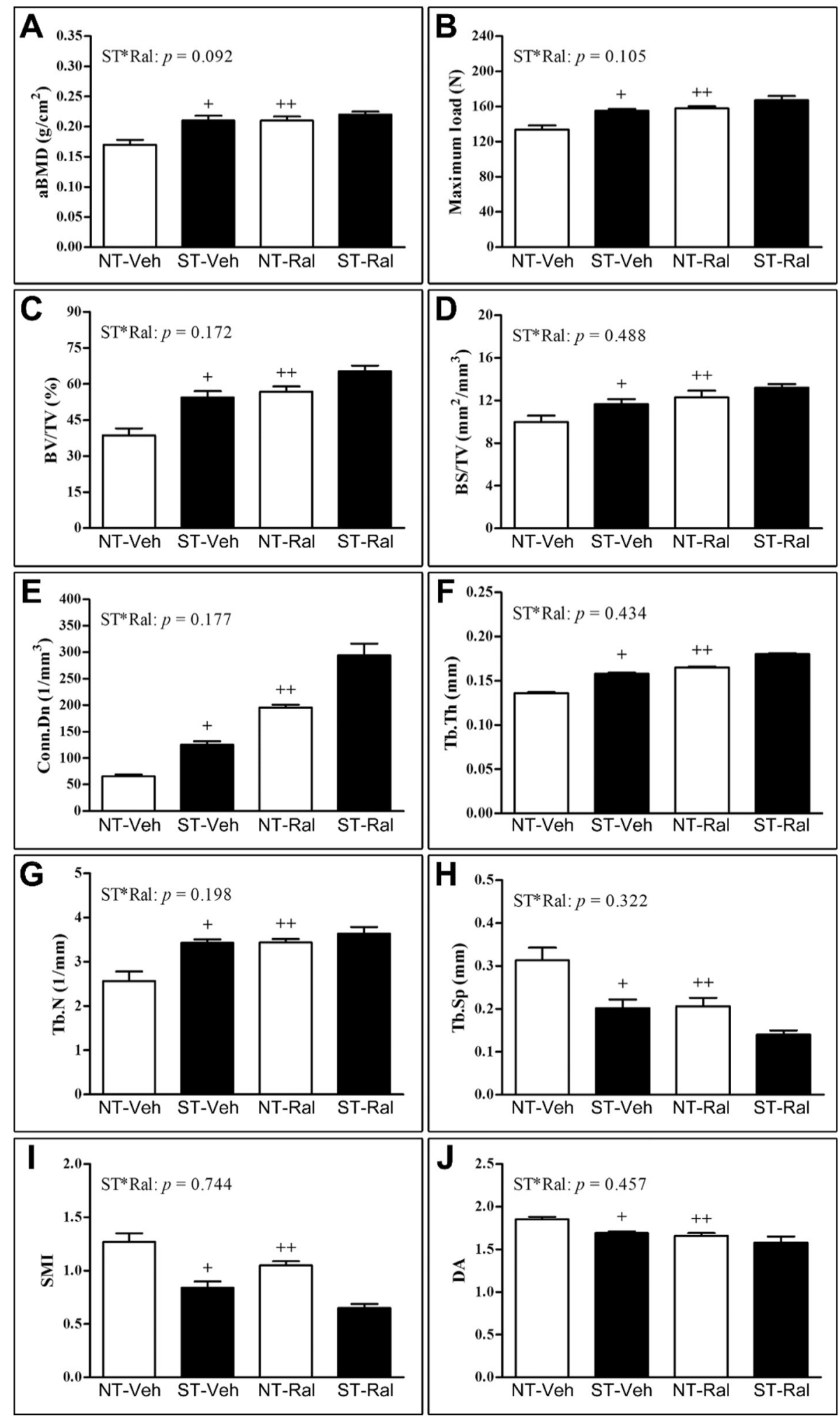

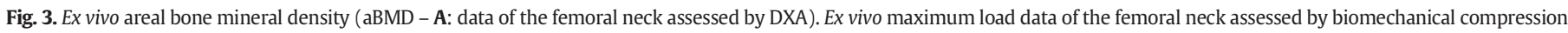

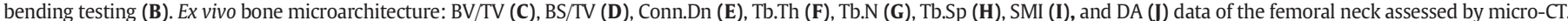

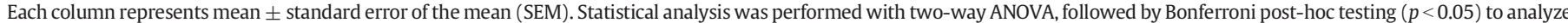

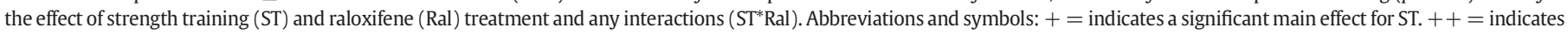

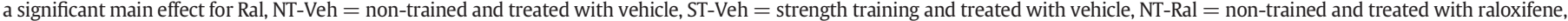
ST-Ral $=$ strength training and treated with raloxifene.

(Lunar DPX Alpha, WI, USA) and software for measuring BMD in small animals. The equipment was calibrated according to manufacturer instructions. The same investigator analyzed all scans. For analysis of the femoral neck, a region of interest (ROI), was identified using a square with a known area $\left(0.72 \mathrm{~mm}^{2}\right)$, which was positioned in the femoral neck region of all specimens. 


\subsection{Biomechanical compression bending testing}

The biomechanical properties of the femur were assessed by the compression test, using a Universal Testing Machine (DL 3000, EMIC $®$, São José dos Pinhais, PR, Brazil). Each femur was placed in a metallic apparatus and maintained vertically (long axis). Load was applied to the area of the femoral head whose vector line of action force is parallel to the long axis of the femur, causing bending moment in the femoral neck region. The deformation rate was $5 \mathrm{~mm} / \mathrm{min}$ with standardized parameters of loaded cells to $2000 \mathrm{~N}$ of capacity [34] until the bone fractured. The load and displacement of the machine crossbar was monitored and recorded using the device software.

\subsection{Microtomography}

Microtomography (micro-computed tomography [CT]) of the femurs was performed using a SkyScan 1172 device (SkyScan, Belgium) at $50 \mathrm{kV}$ and $800 \mathrm{~mA}$. Each femur was positioned in the craniocaudal orientation to obtain slices with a resolution of $12 \times 12 \times 12 \mu \mathrm{m}$ [35]. The images were imported into NRecon software (SkyScan, Leuven, Belgium) and converted from gray scale into Digital Imaging and Communications in Medicine (DICOM) format. To obtain the trabecular bone of the femoral neck, we quantified the volume of interest (VOI), of three-dimensional (3D) structures of trabecular bone in the femoral neck of all animals and extracted the measurements from each dataset in CT-Analyzer (SkyScan, Leuven, Belgium) software (Fig. 2A and B). The internal margin of the cortical bone surrounding the trabecular bone of the femoral neck was defined using the polygon selection tool. The interpolation tool was used on the selected region of trabecular bone. Trabecular bone of the femoral neck is limited by cortical bone in the mediolateral direction. Therefore, greater fidelity for the selected area was acquired using the dynamic interpolation tool. Trabecular bone was selected and inspected using the binary images tool to ensure the use of appropriate threshold values, which were used for all subsequent morphometric analyses. Image processing was required for 3D analysis of the bone morphometric parameters that influence mechanical and structural properties. These parameters were calculated using the CT-Analyzer (SkyScan, Leuven, Belgium) 3D software based on a volume model. Segmentation of each femoral neck was performed using the same software.

The abbreviations below were used for 3D bone morphometric analysis [36]. The measurement parameters of micro-CT analyses were: bone volume fraction (BV/TV; \%), specific bone surface $\left(\mathrm{BS} / \mathrm{TV} ; \mathrm{mm}^{2} / \mathrm{mm}^{3}\right.$ ), trabecular thickness (Tb.Th; $\mathrm{mm}$ ), trabecular number (Tb.N; $1 / \mathrm{mm}$ ), trabecular separation (Tb.Sp; $\mu \mathrm{m}$ ), structure model index (SMI), degree of anisotropy (DA), and connectivity density (Conn. Dn; $1 / \mathrm{mm}^{3}$ ) [37]. The SMI is an index for evaluating whether trabecular bone is rod-like or plate-like. An SMI value of 0 indicates an ideal plate-like structure, while an SMI value of 3 indicates a rod-like structure; a smaller value means a more plate-like structure. The Conn. Dn is a parameter describing trabecular connectivity, with a higher value denoting greater connectivity [38].

\subsection{Immunohistochemistry}

Immunohistochemical evaluation of the expression of runtrelated transcription factor 2 (RUNX2), osterix (OSX), OPG, OCN, receptor activator of nuclear factor-kappa $B$ ligand (RANKL), and TRAP were performed by fixing specimens in $4 \%$ buffered formaldehyde for $24 \mathrm{~h}$ at room temperature, and decalcifying in 10\% ethylenediaminetetraacetic acid (changed weekly) for 8 weeks. Decalcified samples were processed in conventional manner, embedded in paraffin, and submitted to microtomy ( $3 \mu \mathrm{m}$ thick), so that the sections were performed along the coronal plane of the proximal femur.

Histology slides with samples of all experimental groups were then submitted to indirect immunoperoxidase technique and the following primary antibodies (Santa Cruz Laboratories, CA, USA, or Abcam, MA USA): anti-RUNX2 (1:100; SC8566), anti-OSX (1:200; AB22552), anti-OCN (1:180; SC18319), anti-OPG (1:150; SC11383), anti-RANKL (1:100; SC7627), and anti-TRAP (1:100, SC30832). The dilution of primary antibodies was based on a titration test. Immunohistochemical processing followed the protocol described by Garcia et al. [39].

Histological sections were examined under bright field illumination on a light microscope (Optiphot-2, Nikon, Japan) by investigators who were blind to treatment assignments. Four consecutive areas were analyzed at the central portion of the femoral neck. Each of these areas was $300 \times 400 \mu \mathrm{m}$, comprising a total area of $1200 \times 400 \mu \mathrm{m}, 0.48 \mathrm{~mm}^{2}$. Immunolabeling was defined as a brownish staining present in the nuclear (RUNX2, OSX) or cytosolic (OCN, OPG, RANKL, and TRAP) cell compartments. The scores for RUNX2, OSX, and TRAP immunolabeling pattern were modified from Faria et al. [40]: score 3 indicates high pattern, over 8 immunoreactive (IR) cells per area; score 2 indicates moderate pattern, 3 to 7 IR cells per area; score 1 indicates low pattern, less than 3 IR cells per area; and score 0 indicates the absence of immunolabeling. Only multinucleated TRAP-positive cells near a bone surface were quantified. The scores for OCN, OPG, and RANKL
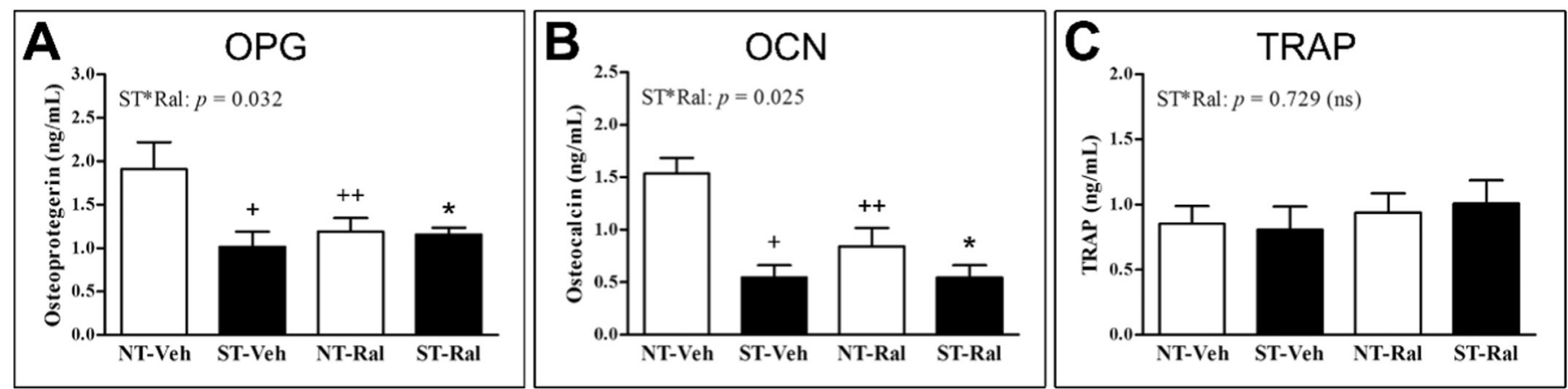

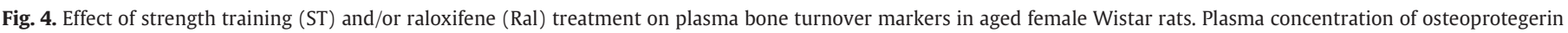

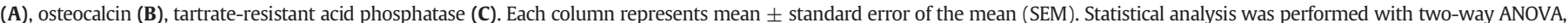

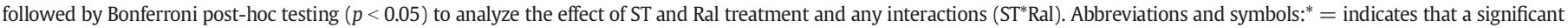

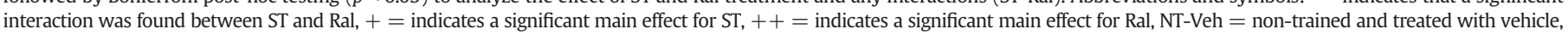
ST-Veh = strength training and treated with vehicle, NT-Ral = non-trained and treated with raloxifene, ST-Ral = strength training and treated with raloxifene. 
immunolabeling patterns were adapted from Garcia et al. [39]: score 3 , high pattern, approximately $75 \%$ of IR cells per area; score 2 , moderate pattern, approximately $50 \%$ IR cells per area; score 1 , low pattern, approximately $25 \%$ IR cells per area; and score 0 , absence of immunolabeling. These immunolabeling scores were compared among experimental groups.

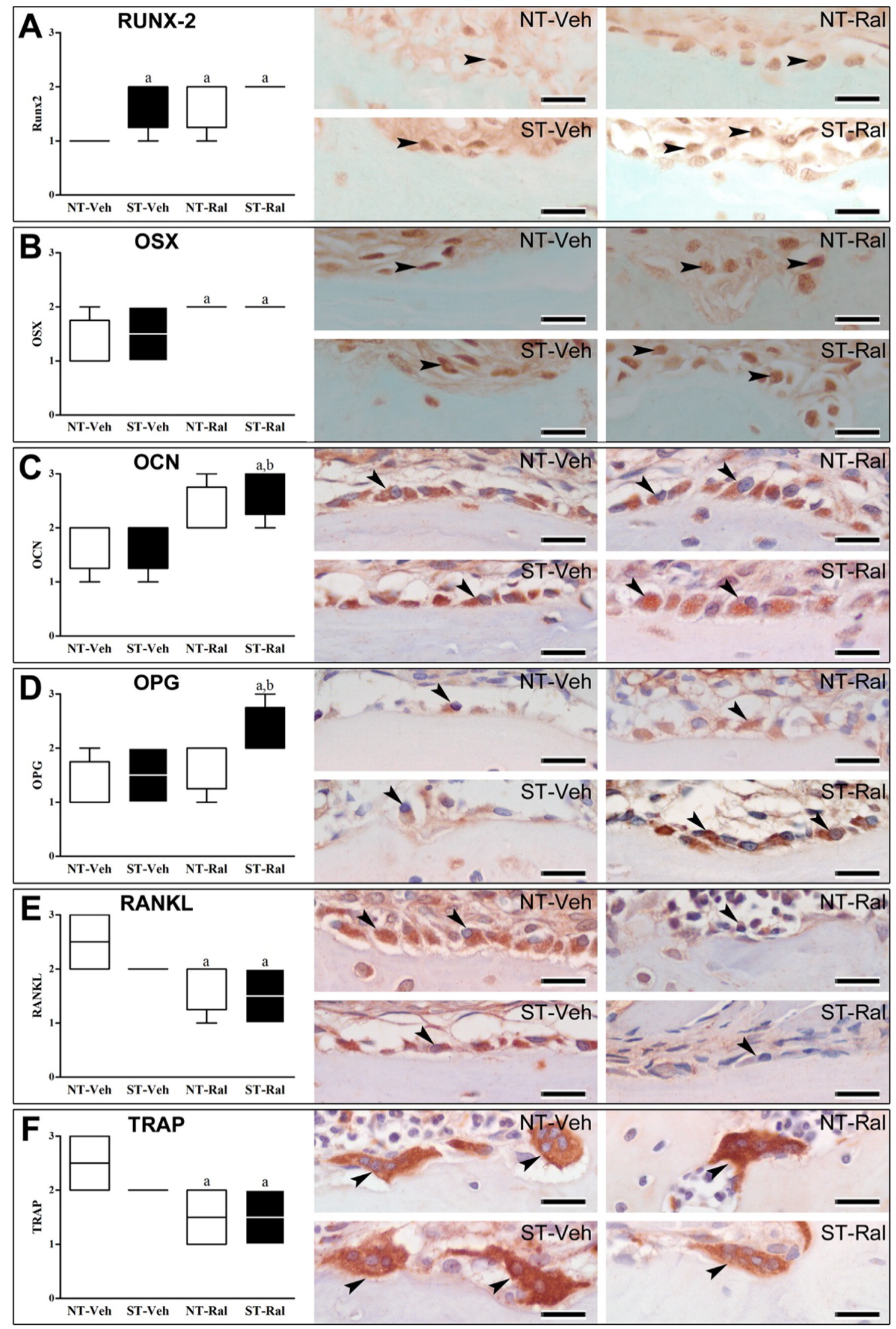




\subsection{Statistical analysis}

All data are presented as means \pm standard errors of the mean (SEM). Significant differences were determined by two-way analysis of variance (ANOVA) followed by Bonferroni post-hoc tests to identify significant differences between two variables in multiple groups. Significant differences in immunohistochemical analyses were identified by the nonparametric Kruskal-Wallis test followed by the Newman-Keuls post-hoc test. For all analyses, $p<0.05$ was considered statistically significant. Statistical analyses were conducted using SigmaPlot v12 (SYSTAT, San Jose, CA, USA) and Biostat V5.3 for immunohistochemical analyses. All animal groups comprised ten animals.

\section{Results}

\subsection{Effect of ST and Ral treatment on body weight, uterus weight, and estradiol plasma concentration}

After the 120-day intervention, there were no significant main effects for Ral or ST, and no significant interactions between Ral and ST for body weight ( $p>0.05$, Table 1$)$. Successful OVX was confirmed at sacrifice, using uterus weight and estradiol plasma concentration. There were no significant effects for ST or Ral, and no significant interactions between ST and Ral for uterus weight and estradiol plasma concentration $(p>0.05$, Table 1$)$.

\subsection{Effect of ST and Ral treatment on bone mass, strength, and trabecular microarchitecture in the femoral neck}

The representative 3D reconstructed micro-CT images of the trabecular femoral neck bone from each treatment group are shown in Fig. 2 (C-F).

Fig. 3 shows the impact of ST and Ral treatment on bone mass, strength, and trabecular microarchitecture in the femoral neck of aged female Wistar rats. Animals in the ST-Veh group had significantly higher $\operatorname{aBMD}(p=0.001)$, maximum load $(p=0.001), \operatorname{BV} / \mathrm{TV}(p=$ $0.001), \mathrm{BS} / \mathrm{TV}(p=0.023)$, Conn.Dn $(p=0.001)$, Tb.Th $(p=0.001)$, and Tb.N $(p=0.012)$, and lower SMI $(p=0.001)$, Tb.Sp $(p=0.001)$, and DA ( $p=0.002)$, compared with the NT-Veh group. In the NT-Ral group, Ral treatment for 16 weeks significantly improved bone strength, as indicated by an increase in maximum load ( $p=0.001)$, bone mass (aBMD, $p=0.004)$, and parameters of bone microarchitecture, including $\mathrm{BV} / \mathrm{TV}(p=0.001), \mathrm{BS} / \mathrm{TV}(p=0.002)$, Conn.Dn $(p=0.001)$, Tb.N ( $p=0.011)$, and Tb.Th $(p=0.001)$, compared with the NTVeh group. In this group, the Ral treatment was coupled with decreases in SMI $(p=0.002)$, trabecular separation $(p=0.001)$, and DA ( $p=0.007$ ), compared with the NT-Veh group. The femoral neck from age- and OVX-related deterioration was positively affected by ST and Ral treatment in an independent manner.

\subsection{Effect of ST and Ral treatment on plasma bone turnover markers}

A summary of the plasma bone biomarker levels assessed by ELISA in each treatment group is shown in Fig. 4. ST (ST-Veh) and Ral (NT-Ral) significantly reduced the plasma levels of OPG ( $p=0.003$ and $p=0.001)$ and OCN ( $p=0.014$ and $p=0.002)$, respectively, compared with NT-Veh. Two-way ANOVA showed a significant interaction between ST and Ral in relation to OPG $\left(\mathrm{F}_{(1,23)}=5.214 ; p=0.032\right)$ and OCN $\left(\mathrm{F}_{(1,25)}=5.652 ; p=0.025\right)$. The plasma concentration of TRAP, a biochemical marker of bone resorption, was not affected by ST and Ral treatment; there was no significant interaction between these two variables.

\subsection{Effect of ST and Ral treatment on bone tissue biomarkers}

The immunohistochemical method used to detect RUNX2, OSX, OCN, OPG, RANKL, and TRAP showed high specificity for these proteins, which was confirmed by the complete absence of immunolabeling in the negative control. Immunolabeling for RUNX2 and OSX was particularly apparent in the nuclear compartment of pre-osteoblasts. Immunolabeling for OCN, OPG, and RANKL was predominantly expressed in the cytosol of osteoblasts. Immunolabeling for TRAP was predominantly expressed in multinucleated osteoclasts. The results of immunohistochemical analyses and the pattern of immunolabeling for all biomarkers are shown in Fig. 5.

RUNX2, a transcription factor that regulates osteoblastogenesis, showed higher immunolabeling in ST-Veh $(p=0.0105)$, NT-Ral $(p=0.0105)$, and ST-Ral $(p=0.0006)$ groups, compared with the NT-Veh group. However, Ral treatment, with or without ST, promoted higher immunolabeling to OSX ( $p=0.0105)$, compared with the NT-Veh group.

For the main local regulators of osteoclastogenesis and osteoclast activity, RANKL and OPG, we found that the ST-Ral group showed decreased immunolabeling of RANKL $(p=0.004)$ and increased immunolabeling of OPG ( $p=0.0034)$, compared with the NT-Veh group. Additionally, immunolabeling of RANKL was less in the NT-Ral ( $p=0.033$ ) group compared with the NT-Veh group. The immunolabeling for TRAP, an osteoclastic biomarker, followed the same pattern of immunolabeling as RANKL; the NT-Ral ( $p=0.0056)$ and ST-Ral $(p=0.0056)$ groups had fewer TRAP-positive cells compared with the NT-Veh group. A marker of mature osteoblast activity, OCN, showed greater immunolabeling in the ST-Ral $(p=0.0024)$ group compared with the NT-Veh group.

\section{Discussion}

This study clearly demonstrates that ST and Ral can prevent deterioration of bone mineral density, microarchitecture, and strength in the femoral neck of aged estrogen-deficient Wistar female rats. This shows that the critical role of estrogen deficiency in bone loss is attenuated to the same extent by ST and Ral.

Femoral neck fractures cause the most morbidity and mortality of any osteoporotic fracture. As such, a study investigating potential therapeutic interventions for the prevention of bone loss due to osteoporosis is highly relevant [41]. However, most studies evaluate the femur, the vertebrae, and the tibia of young adult OVX rats [42-45]. Therefore, appropriate experimental design is crucial for enabling adequate analysis of postmenopausal bone loss. Performing ovariectomy in the 14th month enables study of a subject with a mature skeleton [46] that has undergone a change in the estrous cycle, and is therefore an appropriate animal model for primary osteoporosis. To the best of our knowledge, this is the first report on the effects of Ral and ST on the femoral neck of aged rats, as evaluated by micro-CT, aBMD, maximum load, and immunolabeling of important biomarkers of bone metabolism.

Studies with premenopausal [47] and postmenopausal women [48] demonstrate the influence of hormonal profile and physical exercise on BMD. Bassey et al. [47] showed that after high impact exercise,

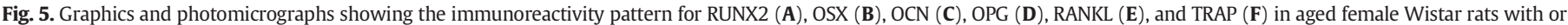

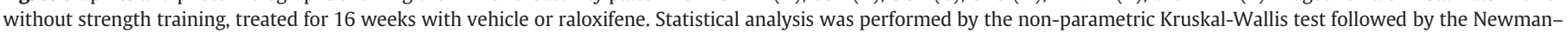

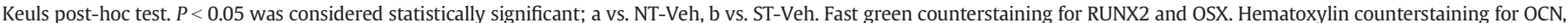

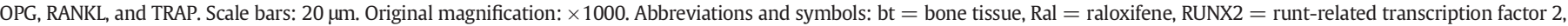

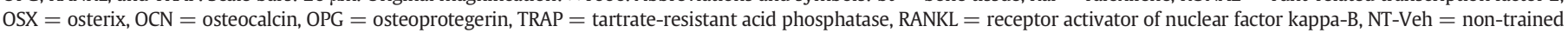

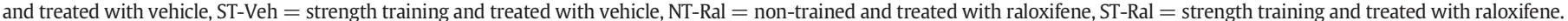


premenopausal women had significantly increased femoral BMD, while postmenopausal women did not. Wågert et al. [48] evaluated proximal femur, lumbar spine, and total body BMD in three postmenopausal women who performed jumping exercises with or without Ral. These authors showed that exercise without Ral reduced values for trochanteric BMD; however, when Ral was added, these values increased. Thus, this study did not show significant differences in femoral neck BMD when exercise was performed even with Ral.

In the present study, we demonstrated that ST and Ral interventions led to the decrease of osteoporosis factors and increase of bone quality of the femoral neck of aged estrogen-deficient Wistar rats. Ral was an effective treatment, preventing the deterioration of bone mass, strength, and microstructure. Our results are in agreement with a study by Turner et al. [49], which showed an increase in BMD and bone strength in the femoral neck of OVX rats (Sprague-Dawley) after Ral treatment. Ral has also been shown to improve bone microarchitecture by increasing BV/TV, Tb.N, and Tb.Th, and decreasing SMI and Tb.S in female C57BL/ 6 mice [31]. The ability of Ral to improve bone parameters can be explained by the ability to stimulate the genesis and activity of osteoblasts and reduce the genesis and activity of osteoclasts. We found that treatment with Ral increases immunolabeling of the transcriptional factors RUNX2 and OSX, demonstrating that the therapy may have stimulated osteoblastogenesis. In addition, Ral decreased the amount of osteoclasts (TRAP-positive cells) via downregulation of RANKL and upregulation of OPG in femoral neck bone. These findings are in accordance with Luvizuto et al. [32], who demonstrated the decrease in RANKL immunolabeling in alveolar bone repair in females. Furthermore, in this study, we show that serum markers of bone turnover OCN and OPG were decreased in the NT-Ral group compared to the NT-Veh group. It is important to note that compared with premenopausal women, levels of both bone formation and resorption markers are substantially higher in postmenopausal women, and that the higher state of bone turnover is maintained for many years after menopause [50]. Together with evidence from a study on postmenopausal women receiving long-term treatment with Ral, which led to a lower level of OCN [51], we have demonstrated that in aged OVX female rats, assessment of plasma OCN and OPG can be used to monitor treatment effectiveness.

The training program also induced improvements in bone mass, microarchitecture, and strength. An interesting finding in this study is that ST with a gradual increase in load was able to affect trabecular bone parameters of aged OVX rats in a manner similar to pharmacological therapy with Ral. The mechanical properties of trabecular bone depend not only on the amount of bone tissue, but also on its microarchitecture, which arises from the activity of bone cells. There is a lack of studies examining the effects of exercise on bone metabolism of aged rats. Data from Tromp et al. [42] showed that the effects of mechanical loading on the trabecular bone of the femoral head in Wistar OVX young rats were not significant. Lespessailles et al. [52] showed that running in 6-month-old female Wistar rats did not prevent trabecular bone loss induced by an ovariectomy. In contrast, with these findings and in agreement with our study, Murai et al. [45] and Renno et al. [44] showed that femoral BMD and bone strength increased in adult female OVX Wistar rats that performed exercise training. Our results agree with those of Bonnet et al. [43], who found that treadmill exercise in female Wistar OVX rats resulted in an increase in BV/TV, Tb.N, and Tb.Th, compared with OVX rats that did not exercise. Thus, our results suggest that the benefits of ST can be explained by their ability to exert a positive effect on osteoblastogenesis, via an increase in the immunolabeling of RUNX2. Furthermore, we found that plasma OCN and OPG were significantly higher in the NT-Veh group than in the STVeh group. This indicated that an ovariectomy causes high bone turnover, which can be reversed with ST, and that these two markers are a potential basis for monitoring the effectiveness of treatment.

This study showed that both ST and Ral result in a reduction of the SMI and DA. The implication of a lower SMI is particularly relevant to studies on osteoporosis in aging females, as it is indicative of more favorable conditions for transition from plate-like to rod-like architecture. Lower SMI values suggest a more plate-like structure and improved bone strength. Furthermore, the DA is inversely correlated with bone mass and positively correlated with the risk of fracture [53], with higher values indicating higher anisotropy [54]. These results also highlighted ST and Ral as a class of antiaging therapeutics that may promote bone formation and reduce bone loss associated with hypoestrogenism.

ST and Ral showed no interactions in terms of bone microarchitecture, mass, or strength. Other studies investigating potential interactions with ST have not provided any evidence for interactions. Murai et al. [45] showed that creatine supplementation with exercise training did not result in an improvement in bone strength, mass, or histomorphometric parameters in OVX rats. Lespessailles et al. [52] showed that running plus infusion of zoledronic acid had no additive effects on femur bone health. Immunohistochemical analysis showed that treatment with ST and Ral stimulated osteoblastogenesis while inhibiting osteoclastogenesis. In addition, treatment increased osteoblastic activity, as evidenced by higher OCN immunolabeling in the femoral neck. Osteoclastic activity was also negatively modulated, verified by the least amount of TRAP-positive osteoclasts, less immunolabeling for RANKL, and greater immunolabeling for OPG. However, plasma bone biomarkers showed an interaction in the ST-Ral group; the plasma OCN and OPG were significantly lower in this group compared with the NT-Veh group, and were inversely correlated with the tissue OPG and OCN biomarkers, showing that when levels of plasma bone markers decrease, the levels of tissue bone markers increase. This finding is particularly interesting because it again allows us to use these markers as predictors of effectiveness of treatment. Our study indicates that ST and Ral interact with each other; therefore, we are conducting further studies involving gene expression and protein quantification to analyze this potential interaction.

There are some limitations. The present study explored the effects of ST and Ral in aged OVX female rats. These interventions may not confer the same benefits for women with hypoestrogenism. Therefore, future clinical studies are needed to validate these interventions in women. Another critical point is that ST was able to prevent osteoporosisinduced hypoestrogenism as much as Ral, but as a sole treatment in the prevention of osteoporosis, it should be prescribed and monitored by health professionals to ensure that achievement and frequency of training are carried out satisfactorily.

\section{Conclusion}

We conclude that hypoestrogenism-induced osteopenia during the aging process can be reversed using ST and Ral. Therefore, we suggest that physical exercise (ST) and drug treatment (Ral) are effective interventions in the prevention of osteopenia. They induce an improvement in bone quality, along with a lower risk of fractures, and consequently a better quality of life for women in whom hormone replacement therapy or bisphosphonates are contraindicated. Moreover, ST itself, without associated drug therapy, was able to improve bone health in a manner similar to Ral. Therefore, exercise that promotes muscle strength could be considered as a therapeutic treatment for primary osteoporosis.

\section{Grants}

CAPES (Coordination of Improvement of Higher Education Personnel/ number 2010/03,112-6) and FAPESP (Fundação de Amparo à Pesquisa do Estado de São Paulo/number 2010/09,393-7) supported this work.

\section{Disclosures}

There are no conflicts of interest to be disclosed by any author. 


\section{Acknowledgments}

We thank the Universidade Estadual Paulista "Júlio de Mesquita Filho," research foundations (Fundação de Amparo à Pesquisa do Estado de São Paulo - FAPESP and Coordenação de Aperfeiçoamento de Pessoal de Nível Superior - CAPES), and the Brazilian Society of Physiology for supporting the present study. We would also like to thank Dr. Euclides Braga Malheiros (UNESP, Jaboticabal, SP, Brazil) and Dr. Sílvia Helena Venturoli Perri (Faculty of Veterinary Medicine, UNESP, Araçatuba, SP, Brazil) for the statistical design of the study, and Dr. Sergio Diniz Garcia (Faculty of Veterinary Medicine, UNESP, Araçatuba, SP, Brazil) for monitoring and helping with the care of animals during the aging process.

\section{Appendix A. Supplementary data}

Supplementary data to this article can be found online at http://dx. doi.org/10.1016/j.bone.2015.11.023.

\section{References}

[1] E.S. Christenson, X. Jiang, R. Kagan, P. Schnatz, Osteoporosis management in postmenopausal women, Minerva Ginecol. 64 (3) (2012) 181-194.

[2] C. Cooper, Z.A. Cole, C.R. Holroyd, S.C. Earl, N.C. Harvey, E.M. Dennison, L.J. Melton, S.R. Cummings, J.A. Kanis, Secular trends in the incidence of hip and other osteoporotic fractures, Osteoporos. Int. 22 (5) (2011) 1277-1288.

[3] L. Lanyon, T. Skerry, Postmenopausal osteoporosis as a failure of bone's adaptation to functional loading: a hypothesis, J. Bone Miner. Res. 16 (11) (2001) 1937-1947.

[4] S.R. Cummings, L.J. Melton, Epidemiology and outcomes of osteoporotic fractures, Lancet 359 (9319) (2002) 1761-1767.

[5] J.A. Cauley, D. Chalhoub, A.M. Kassem, G.E.H. Fuleihan, Geographic and ethnic disparities in osteoporotic fractures, Nat. Rev. Endocrinol. 10 (6) (2014) 338-351.

[6] K.E. Ensrud, Epidemiology of fracture risk with advancing age, J. Gerontol. A Biol. Sci. Med. Sci. 68 (10) (2013) 1236-1242.

[7] J.R. Rey, E.V. Cervino, M.L. Rentero, E.C. Crespo, A.O. Alvaro, M. Casillas, Raloxifene: mechanism of action, effects on bone tissue, and applicability in clinical traumatology practice, Open Orthop. J. 3 (2009) 14-21.

[8] D.S. Buist, A.Z. LaCroix, D. Manfredonia, T. Abbott, Identifying postmenopausal women at high risk of fracture in populations: a comparison of three strategies, J. Am. Geriatr. Soc. 50 (6) (2002) 1031-1038.

[9] ESHRE Capri Workshop Group, Bone fractures after menopause, Hum. Reprod. Update 16 (6) (2010) 761-773.

[10] H.B. Jia, J.X. Ma, X.L. Ma, J.T. Yu, R. Feng, L.Y. Xu, J. Wang, D. Xing, S.W. Zhu, Y. Wang, Estrogen alone or in combination with parathyroid hormone can decrease vertebra MEF2 and sclerostin expression and increase vertebral bone mass in ovariectomized rats, Osteoporos. Int. 25 (12) (2014) 2743-2754.

[11] T. Miyazaki, F. Tokimura, S. Tanaka, A review of denosumab for the treatment of osteoporosis, Patient Prefer. Adherence 8 (2014) 463-471.

[12] S. Canpolat, N. Tug, A.D. Seyran, S. Kumru, B. Yilmaz, Effects of raloxifene and estradiol on bone turnover parameters in intact and ovariectomized rats, J. Physiol. Biochem. 66 (1) (2010) 23-28.

[13] J. Reginster, H.W. Minnie, O.H. Sorensen, M. Hooper, C. Roux, M.L. Brandi, B. Lund, D. Ethgen, S. Pack, I. Roumagnac, R. Eastell, Randomized trial of the effects of risedronate on vertebral fractures in women with established postmenopausal osteoporosis. vertebral efficacy with risedronate therapy (VERT) Study Group, Osteoporos. Int. 11 (1) (2000) 83-91.

[14] S. Kato, Molecular mechanism of tissue-specific actions of SERM, Clin. Calcium 16 (9) (2006) 1469-1474.

15] S. Martino, J.A. Cauley, E. Barrett-Connor, T.J. Powles, J. Mershon, D. Disch, R. Secrest, S.R. Cummings, C.O.R.E. Investigators, Continuing outcomes relevant to evista: breast cancer incidence in postmenopausal osteoporotic women in a randomized trial of raloxifene, J. Natl. Cancer Inst. 96 (23) (2004) 1751-1761.

[16] B. Ettinger, D.M. Black, B.H. Mitlak, R.K. Knickerbocker, T. Nickelsen, H.K. Genant, C. Christiansen, P.D. Delmas, J.R. Zanchetta, J. Stakkestad, C.C. Glüer, K. Krueger, F.J. Cohen, S. Eckert, K.E. Ensrud, L.V. Avioli, P. Lips, S.R. Cummings, Reduction of vertebral fracture risk in postmenopausal women with osteoporosis treated with raloxifene: results from a 3-year randomized clinical trial. multiple outcomes of raloxifene evaluation (MORE) investigators, JAMA 282 (7) (1999) 637-645.

[17] H. Matsumori, K. Hattori, H. Ohgushi, Y. Dohi, Y. Ueda, H. Shigematsu, N. Satoh, H. Yajima, Y. Takakura, Raloxifene: its ossification-promoting effect on female mesenchymal stem cells, J. Orthop. Sci. 14 (5) (2009) 640-645.

[18] A. Taranta, M. Brama, A. Teti, V. De luca, R. Scandurra, G. Spera, D. Agnusdei, Termine JD, S. Migliaccio, The selective estrogen receptor modulator raloxifene regulates osteoclast and osteoblast activity in vitro, Bone 30 (2) (2002) 368-376.

[19] T. Notomi, S.J. Lee, N. Okimoto, et al., Effects of resistance exercise training on mass, strength, and turnover of bone in growing rats, Eur. J. Appl. Physiol. 82 (4) (2000) 268-274.
[20] T. Notomi, Y. Okazaki, N. Okimoto, S. Saitoh, T. Nakamura, M. Suzuki, A comparison of resistance and aerobic training for mass, strength and turnover of bone in growing rats, Eur. J. Appl. Physiol. 83 (6) (2000) 469-474.

[21] L. Maïmoun, C. Sultan, Effect of physical activity on calcium homeostasis and calciotropic hormones: a review, Calcif. Tissue Int. 85 (4) (2009) 277-286.

[22] W.R. Thompson, C.T. Rubin, J. Rubin, Mechanical regulation of signaling pathways in bone, Gene 503 (2) (2012) 179-193.

[23] B.L. Riggs, S. Khosla, L.J. Melton III, Sex steroids and the construction and conservation of the adult skeleton, Endocr. Rev. 23 (3) (2002) 279-302.

[24] L.K. Saxon, G. Galea, L. Meakin, J. Price, L.E. Lanyon, Estrogen receptors $\alpha$ and $\beta$ have different gender-dependent effects on the adaptive responses to load bearing in cancellous and cortical bone, Endocrinology 153 (5) (2012) 2254-2266.

[25] J.I. Aguirre, L.I. Plotkin, A.R. Gortazar, et al., A novel ligand-independent function of the estrogen receptor is essential for osteocyte and osteoblast mechanotransduction, J. Biol. Chem. 282 (35) (2007) 25501-25508.

[26] K. Lee, H. Jessop, R. Suswillo, G. Zaman, L. Lanyon, Endocrinology: bone adaptation requires oestrogen receptor-alpha, Nature 424 (6947) (2003) 389

[27] J. LeFevre, M.K. McClintock, Reproductive senescence in female rats: A longitudinal study of individual differences in estrous cycles and behavior, Biol. Reprod. 38 (4) (1988) 780-789.

[28] G.E. Besteti, M.J. Reymond, F. Blanc, C.E. Boujon, B. Furrer, G.L. Rossi, Functional and morphological changes in the hypothalamo-pituitary-Gonadal Axis of aged female rats, Biol. Reprod. 45 (2) (1991) 221-228.

[29] D.W. Matt, J. Lee, P.L. Sarver, H.L. Judd, J.K. Lu, Chronological changes in fertility, fecundity and steroid hormone secretion during consecutive pregnancies in aging rats, Biol. Reprod. 34 (3) (1986) 478-487.

[30] H.B. Waynforth (Ed.), Experimental and Surgical Techniques in the Rat, Academic Press, New York, 1980.

[31] A. Cano, S. Dapía, I. Noguera, B. Pineda, C. Hermenegildo, R. del Val, J.R. Caeiro, M.A. García-Pérez, Comparative effects of 17beta-estradiol, raloxifene and genistein on bone 3D microarchitecture and volumetric bone mineral density in the ovariectomized mice, Osteoporos. Int. 19 (6) (2008) 793-800.

[32] E.R. Luvizuto, T.P. Queiroz, Dias SM, et al., Histomorphometric analysis and immunolocalization of RANKL and OPG during the alveolar healing process in female ovariectomized rats treated with oestrogen or raloxifene, Arch. Oral Biol. 55 (1) (2010) 52-59.

[33] T.A. Hornberger Jr., R.P. Farrar, Physiological hypertrophy of the FHL muscle following 8 weeks of progressive resistance exercise in the rat, Can. J. Appl. Physiol. 29 (1) (2004) 16-31.

[34] C.H. Turner, D.B. Burr, Basic biomechanical measurements of bone: a tutorial, Bone 14 (4) (1993) 595-608.

[35] S. Tatsumi, M. Ito, Y. Asaba, K. Tsutsumi, K. Ikeda, Life-long caloric restriction reveals biphasic and dimorphic effects on bone metabolism in rodents, Endocrinology 149 (2) (2008) 634-641

[36] A.M. Partiff, M.K. Drezner, Glorieux FH, et al., Bone histomorphometry: standardization of nomenclature, symbols and units, J. Bone Miner. Res. 2 (6) (1987) 595-610.

[37] M.L. Bouxsein, S.K. Boyd, B.A. Christiansen, R.E. Guldberg, K.J. Jepsen, R. Müller, Guidelines for assessment of bone microstructure in rodents using microcomputed tomography, J. Bone Miner. Res. 25 (7) (2010) 1468-1486.

[38] A. Odgaard, H.J. Gundersen, Quantification of connectivity in cancellous bone, with special emphasis on 3D reconstructions, Bone 14 (2) (1993) 173-182.

[39] V.G. Garcia, M. Longo, E.C. Gualberto Júnior, A.F. Bosco, M.J. Nagata, E. Ervolino, L.H. Theodoro, Effect of the concentration of phenothiazine photosensitizers in antimicrobial photodynamic therapy on bone loss and the immune inflammatory response of induced periodontitis in rats, J. Periodontal Res. 49 (5) (2014) 584-594.

[40] P.E. Faria, R. Okamoto, R.M. Bonilha-Neto, S.P. Xavier, A.C. Santos, L.A. Salata, Immunohistochemical, tomographic and histological study on onlay iliac grafts remodeling, Clin. Oral Implants Res. 19 (4) (2008) 393-401.

[41] M. Almeida, C.A. O'Brien, Basic biology of skeletal aging: role of stress response pathways, J. Gerontol. A Biol. Sci. Med. Sci. 68 (10) (2013) 1197-1208.

[42] A.M. Tromp, N. Bravenboer, E. Tanck, et al., Additional weight bearing during exercise and estrogen in the rat: the effect on bone mass, turnover, and structure, Calcif. Tissue Int. 79 (6) (2006) 404-415.

[43] N. Bonnet, H. Beaupied, L. Vico, E. Dolleans, N. Laroche, D. Courteix, C.L. Benhamou, Combined effects of exercise and propranolol on bone tissue in ovariectomized rats, J. Bone Miner. Res. 22 (4) (2007) 578-588.

[44] A.C. Renno, A.R. Silveira Gomes, R.B. Nascimento, T. Salvini, N. Parizoto, Effects of a progressive loading exercise program on the bone and skeletal muscle properties of female osteopenic rats, Exp. Gerontol. 42 (6) (2007) 517-522.

[45] I.H. Murai, H. Roschel, L.V. Pabis, et al., Exercise training, creatine supplementation, and bone health in ovariectomized rats, Osteoporos. Int. 26 (4) (2015) 1395-1404.

[46] P.P. Lelovas, T.T. Xanthos, S.E. Thoma, G.P. Lyritis, I.A. Dontas, The laboratory rat as an animal model for osteoporosis research, Commun. Med. 58 (5) (2008) 424-430.

[47] E.J. Bassey, M.C. Rothwell, J.J. Littlewood, D.W. Pye, Pre- and postmenopausal women have different BoneMineral density responses to the SameHigh-impact exercise, J. Bone Miner. Res. 13 (12) (1998) 1805-1813.

[48] Wågert P. Von Heideken, H. Littbrand, A. Johansson, P. Nordström, Y. Gustafson, Jumping exercises with and without raloxifene treatment in healthy elderly women, J. Bone Miner. Metab. 20 (6) (2002) 376-382.

[49] C.H. Turner, M. Sato, H.U. Bryant, Raloxifene preserves bone strength and bone mass in ovariectomized rats, Endocrinology 135 (5) (1994) 2001-2005.

[50] M.J. Seibel, Biochemical markers of bone turnover: part I: biochemistry and variability, Clin. Biochem. Rev. 26 (4) (2005) 97-122. 
[51] S. Palomba, F.G. Numis, Mossetti Get, et al., Raloxifene administration in postmenopausal women with osteoporosis: effect of different BsmI vitamin D receptor genotypes. Hum. Reprod. 18 (1) (2003) 192-198.

[52] E. Lespessailles, C. Jaffré, H. Beaupied, et al., Does exercise modify the effects of zoledronic acid on bone mass, microarchitecture, biomechanics, and turnover in ovariectomized rats? Calcif. Tissue Int. 85 (2) (2009) 146-157.
[53] Masako lto, Assessment of bone quality using micro-computed tomography (microCT) and synchrotron micro-CT, J. Bone Miner. Metab. 23 (Suppl) (2005) 115-121.

[54] T. Hildebrand, P. Rüegsegger, Quantification of bone microarchitecture with the structure model index, Comput. Methods Biomech. Biomed. Engin. 1 (1) (1997) $15-23$. 